Document downloaded from:

http://hdl.handle.net/10251/46841

This paper must be cited as:

Romaguera Bonilla, S.; Tirado Peláez, P.; Valero Sierra, O. (2012). Complete partial metric spaces have partially metrizable computational models. International Journal of Computer Mathematics. 89(3):284-290. doi:10.1080/00207160.2011.559229.

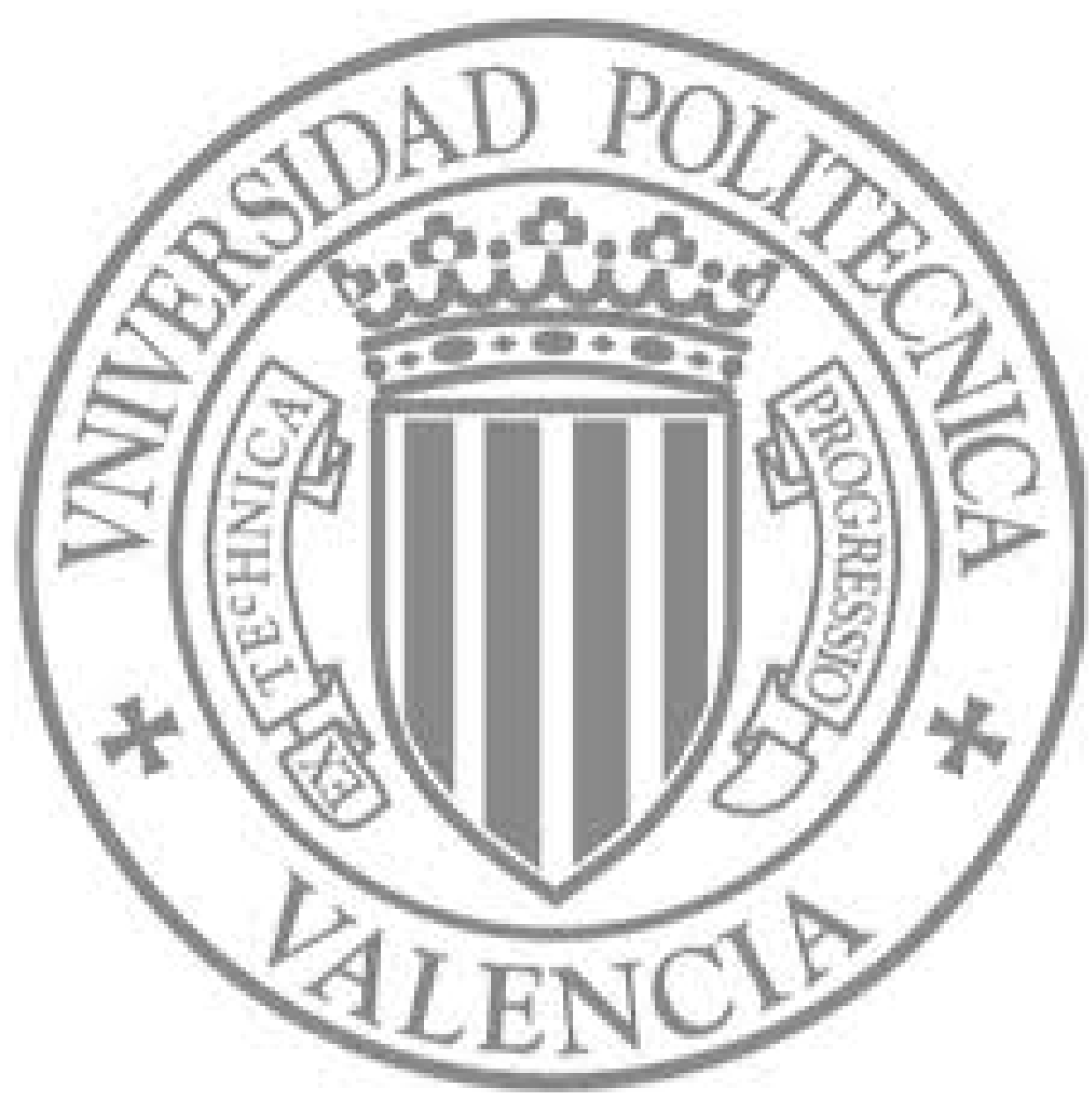

The final publication is available at

http://dx.doi.org/10.1080/00207160.2011.559229

Copyright Taylor \& Francis Ltd 


\title{
Complete partial metric spaces have partially metrizable computational models
}

\author{
S. Romaguera, P. Tirado, O. Valero*
}

\begin{abstract}
We show that the domain of formal balls of a complete partial metric space $(X, p)$ can be endowed with a complete partial metric that extends $p$ and induces the Scott topology. This result, that generalizes well-known constructions of Edalat and Heckmann (Theoret. Comput. Sci. 1998) and Heckmann (Appl. Cat. Struct. 1998) for metric spaces and improves a recent result of Romaguera and Valero (Math. Struct. Comput. Sci. 2009), motivates a notion of a partially metrizable computational model which allows us to characterize those topological spaces that admit a compatible complete partial metric via this model.
\end{abstract}

\section{Introduction}

Motivated by the fact that metric spaces and domain theory constitute fundamental mathematical tools in computer science, several authors have investigated the problem of obtaining links between them and constructing, with this approach, suitable models in the theory of computation $[2,3,5$, 7, 8, 11, 12, 13, 15, 19, 23, etc]. In particular, Edalat and Heckmann [2] presented a very nice and explicit construction of a computational model for (complete) metric spaces by means of the notion of a formal ball. They proved, among other results, that the poset of formal balls of a metric space $(X, d)$ is a domain if and only if $(X, d)$ is complete and that this poset is an $\omega$-domain if and only if $(X, d)$ is complete and separable (previously, Lawson characterized in [11] separable completely metrizable spaces in terms of $\omega$-domains). Later on, Heckmann [7] constructed a partial metric on the (continuous) poset of formal balls of a metric space $(X, d)$ which extends the metric $d$ and induces the Scott topology (similar results were obtained

\footnotetext{
${ }^{*}$ The authors thank the support of the Spanish Ministry of Science and Innovation, under grant MTM2009-12872-C02-01.
} 
by Rutten [19] with a different approach). Heckmann's construction is very interesting because the use of a distance function on the formal balls provides a "quantitative" computational model, which suggested, by one hand, the study of computational models from a quantitative point of view and, on the other hand, the study of partial metrics and related structures by means of domain theory (see $[1,9,14,16,17,18,20,21,22,23$, etc] for recent contributions in these directions).

Here, and improving a recent construction by Romaguera and Valero [16], we shall prove that the domain of formal balls of a complete partial metric space $(X, p)$ can be endowed with a complete partial metric that, among other things, extends the partial metric $p$ and induces the Scott topology. Motivated by this result we shall define the notion of a partially metrizable computational model and then we shall show that the topological spaces having a partially metrizable computational model are exactly those admitting a compatible complete partial metric.

\section{Background}

The letters $\mathbb{N}, \mathbb{R}$ and $\mathbb{R}^{+}$will denote the set of positive integer numbers, the set of real numbers and the set of non-negative real numbers, respectively.

Our basic reference for domain theory is [6], for general topology is [4] and for quasi-metric spaces it is [10].

Let us recall that a partially ordered set, or poset for short, is a set $L$ equipped with a partial order $\sqsubseteq$; it will be denoted by $(L, \sqsubseteq)$ or simply by $L$ if no confusion arises.

A subset $D$ of a poset $L$ is directed provided that it is nonempty and any pair of elements of $D$ has an upper bound in $D$. The least upper bound of a subset $D$ of $L$ is denoted by $\bigsqcup D$ if it exists.

A poset $L$ is said to be directed complete, and is called a dcpo, if every directed subset of $L$ has a least upper bound.

An element $x$ of $L$ is said to be maximal if the condition $x \sqsubseteq y$ implies $x=y$. The set of all maximal points of $L$ will be denoted by $\operatorname{Max}((L, \sqsubseteq))$ or simply by $\operatorname{Max}(L)$ if no confusion arises.

Let $L$ be a poset and $x, y \in L$; we say that $x$ is way below $y$, in symbols $x \ll y$, if for each directed subset $D$ of $L$ for which $\bigsqcup D$ exists, the relation $y \sqsubseteq \bigsqcup D$ implies the existence of some $z \in D$ with $x \sqsubseteq z$.

A poset $L$ is called continuous if for each $x \in L$, the set $\Downarrow x=\{u \in L$ : $u \ll x\}$ is directed and $x=\bigsqcup(\Downarrow x)$. 
A continuous poset which is also a dcpo is called a continuous domain or, simply, a domain.

A subset $B$ of a poset $L$ is a basis for $L$ if for each $x \in L$, the set $\Downarrow x_{B}=\{u \in B: u \ll x\}$ is directed and $x=\bigsqcup\left(\Downarrow x_{B}\right)$.

Recall that a poset has a basis if and only if it is continuous. Therefore, a dcpo has a basis if and only if it is a domain.

A dcpo having a countable basis is said to be an $\omega$-continuous domain, or simply an $\omega$-domain if no confusion arises.

The Scott topology $\sigma(L)$ of a poset $(L, \sqsubseteq)$ is constructed as follows: A subset $U$ of $L$ is open with respect to $\sigma(L)$ provided that: (i) $U=\uparrow U$, where $\uparrow U=\{y \in L: x \sqsubseteq y$ for some $x \in U\}$; (ii) for each directed subset $D$ of $L$ with $\bigsqcup D \in U$, it follows that $D \cap U \neq \emptyset$.

If $D$ is a subset of $L$, we denote by $\sigma(L)_{\mid D}$ the restriction of $\sigma(L)$ to $D$.

If $(L, \sqsubseteq)$ is a continuous poset, then the sets $\Uparrow x, x \in L$, form an open base for $\sigma(L)$, where $\Uparrow x=\{y \in L: x \ll y\}$ (see [6, Proposition II-1.10]).

The lower (or weak) topology of a poset $L$ is the one that has as a subbase the collection of sets of the form $L \backslash \uparrow x, x \in L$, and is denoted by $\omega(L)$. Let us recall that the supremum topology of $\sigma(L)$ and $\omega(L)$ is the Lawson topology of $L$, which is denoted by $\lambda(L)$. If $D$ is a subset of $L$, we denote by $\lambda(L)_{\mid D}$ the restriction of $\lambda(L)$ to $D$.

If $(X, \tau)$ is a $T_{0}$ topological space, then the binary relation $\leq_{\tau}$ defined on $X$ by $x \leq_{\tau} y \Leftrightarrow x \in \operatorname{cl}\{y\}$, is a partial order on $X$ called the specialization order (see, for instance, p. 42 of [6]).

Following the modern terminology, by a quasi-metric on a set $X$ we mean a function $d: X \times X \rightarrow \mathbb{R}^{+}$such that for all $x, y, z \in X:$ (i) $x=y \Leftrightarrow$ $d(x, y)=d(y, x)=0$; (ii) $d(x, z) \leq d(x, y)+d(y, z)$.

A quasi-metric space is a pair $(X, d)$ such that $X$ is a set and $d$ is a quasi-metric on $X$.

Each quasi-metric $d$ on $X$ induces a $T_{0}$ topology $\tau_{d}$ on $X$ which has as a base the family of open balls $\left\{B_{d}(x, r): x \in X, \varepsilon>0\right\}$, where $B_{d}(x, \varepsilon)=$ $\{y \in X: d(x, y)<\varepsilon\}$ for all $x \in X$ and $\varepsilon>0$.

Note that if $(X, d)$ is a quasi-metric space, then the binary relation $\leq_{d}$ defined on $X$ by $x \leq_{d} y \Leftrightarrow d(x, y)=0$, is a partial order on $X$, which coincides with the specialization order of $\left(X, \tau_{d}\right)$.

Given a quasi-metric $d$ on $X$, then the function $d^{-1}$ defined by $d^{-1}(x, y)=$ $d(y, x)$, is also a quasi-metric on $X$, called the conjugate of $d$, and the function $d^{s}$ defined by $d^{s}(x, y)=\max \left\{d(x, y), d^{-1}(x, y)\right\}$ is a metric on $X$.

On the other hand, the notion of a partial metric space, and its equivalent weightable quasi-metric space, was introduced by Matthews in [13] as a part 
of the study of denotational semantics of dataflow networks.

Let us recall that a partial metric on a set $X$ is a function $p: X \times X \rightarrow \mathbb{R}^{+}$ such that for all $x, y, z \in X:(\mathrm{i}) x=y \Leftrightarrow p(x, x)=p(x, y)=p(y, y)$; (ii) $p(x, x) \leq p(x, y)$; (iii) $p(x, y)=p(y, x)$; (iv) $p(x, z) \leq p(x, y)+p(y, z)-$ $p(y, y)$.

If $p$ is a partial metric on $X$ we denote by ker $p$ the subset of $X$ consisting of all points $x \in X$ such that $p(x, x)=0$. Obviously, every metric $d$ on $X$ is a partial metric with $\operatorname{ker} d=X$.

A partial metric space is a pair $(X, p)$ such that $X$ is a set and $p$ is a partial metric on $X$.

Each partial metric $p$ on $X$ induces a $T_{0}$-topology $\tau_{p}$ on $X$ which has as a base the family of open $p$-balls $\left\{B_{p}(x, \varepsilon): x \in X, \varepsilon>0\right\}$, where $B_{p}(x, \varepsilon)=$ $\{y \in X: p(x, y)<\varepsilon+p(x, x)\}$ for all $x \in X$ and $\varepsilon>0$.

We say that a topological space $(X, \tau)$ admits a compatible partial metric if there is a partial metric $p$ on $X$ such that $\tau_{p}=\tau$.

Matthews observed in [13] that each partial metric $p$ on a set $X$ induces a quasi-metric $d_{p}$ on $X$ given by $d_{p}(x, y)=p(x, y)-p(x, x)$ for all $x, y \in X$. Moreover $\tau_{p}=\tau_{d_{p}}$.

If $(X, p)$ is a partial metric space, then the binary relation $\leq_{p}$ on $X$ given by $x \leq_{p} y \Leftrightarrow p(x, y)=p(x, x)$, is a partial order on $X$. Hence $\left(X, \leq_{p}\right)$ is a poset $([7,13])$. Note that in this case one has $\leq_{p}=\leq_{d_{p}}$.

In Definition 5.3 of [13], Matthews also introduced the notion of a complete partial metric and stated, with a slight different terminology, that a partial metric $p$ on a set $X$ is complete if and only if the metric $\left(d_{p}\right)^{s}$ is complete on $X$.

We will say that a partial metric space $(X, p)$ is complete if $p$ is a complete partial metric on $X$.

\section{Partially metrizable computational models}

Recall [2] that if $(X, d)$ is a metric space, then $\mathbf{B} X=X \times \mathbb{R}^{+}$is said to be the set of formal balls of $(X, d)$, and the relation $\sqsubseteq_{d}$ defined on $\mathbf{B} X$ by $(x, r) \sqsubseteq_{d}(y, s) \Leftrightarrow d(x, y) \leq r-s$, is a partial order on $X$. The pair $\left(\mathbf{B} X, \sqsubseteq_{d}\right)$ is called the poset of formal balls of $(X, d)$.

Then, Edalat and Heckmann proved that for any metric space $(X, d)$, the poset of formal balls is continuous, and the mapping $i: X \rightarrow \mathbf{B} X$, given by $i(x)=(x, 0)$, is a homeomorphism between $\left(X, \tau_{d}\right)$ and $\left(\operatorname{Max}(\mathbf{B} X), \sigma(\mathbf{B} X)_{\mid \operatorname{Max}(\mathbf{B} X)}\right)$, and $\sigma(\mathbf{B} X)_{\mid \operatorname{Max}(\mathbf{B} X)}=\lambda(\mathbf{B} X)_{\mid \operatorname{Max}(\mathbf{B} X)}$.

As we mentioned above, Heckmann constructed in [7] a partial metric 
on the set $\mathbf{B} X$ of formal balls of a metric space $(X, d)$, that extends the metric $d$ and such that both the partial order $\sqsubseteq_{d}$ and the Scott topology are induced by it.

The partial metric $P$ on $\mathbf{B} X$, given below, is a slight modification of Heckmann's original construction (see Section 4 of [16]):

$$
P((x, r),(y, s))=\max \{d(x, y),|r-s|\}+r+s,
$$

for all $(x, r),(y, s) \in \mathbf{B} X$.

In Theorem 1 below w extend Heckmann's construction to (complete) partial metric spaces which also provides an improvement of Theorem 4.1 of $[16]$.

To this end, we first recall some pertinent concepts and facts.

The poset of formal balls of a quasi-metric space $(X, d)$ is the poset $\left(\mathbf{B} X, \sqsubseteq_{d}\right)$, where both $\mathbf{B} X$ and the partial order $\sqsubseteq_{d}$ are defined exactly as in the metric case (see $[1,16]$, where quasi-metric and partial metric versions of several results of Edalat and Heckmann were obtained. In particular, it was proved in Theorem 3.1 of [16] that a partial metric space $(X, p)$ is complete if and only if ( $\left.\mathbf{B} X, \sqsubseteq d_{p}\right)$ is a domain ).

According to Waszkiewicz [22], a weak partial metric on a set $X$ is a function $p: X \times X \rightarrow \mathbb{R}^{+}$such that for all $x, y, z \in X:$ (i) $x=y \Leftrightarrow p(x, x)=$ $p(x, y)=p(y, y)$; (ii) $p(x, y)=p(y, x)$; (iii) $p(x, z) \leq p(x, y)+p(y, z)-p(y, y)$.

Heckmann [7] showed that every weak partial metric $p$ on a set $X$ induces a partial metric $p^{\prime}$ on $X$ given by $p^{\prime}(x, y)=\max \{p(x, y), p(x, x), p(y, y)\}$ for all $x, y \in X$.

Now let $(X, p)$ be a partial metric space. Construct a function $P$ : $\mathbf{B} X \times \mathbf{B} X \rightarrow \mathbb{R}^{+}$by

$$
P((x, r),(y, s))=p(x, y)+r+s,
$$

for all $(x, r),(y, s) \in \mathbf{B} X$.

The proof of the following result is straightforward (compare with the first paragraph of 3.2 in [7]).

Proposition 1. For each partial metric space $(X, p)$, the function $P$ constructed above is a weak partial metric on $\mathbf{B} X$.

Therefore, the function $P^{\prime}: \mathbf{B} X \times \mathbf{B} X \rightarrow \mathbb{R}^{+}$defined by

$$
P^{\prime}((x, r),(y, s))=\max \{P((x, r),(y, s)), P((x, r),(x, r)), P((y, s),(y, s))\},
$$


for all $(x, r),(y, s) \in \mathbf{B} X$, is a partial metric on $\mathbf{B} X$.

Note that, in fact,

$$
P^{\prime}((x, r),(y, s))=\max \{p(x, y)+r+s, p(x, x)+2 r, p(y, y)+2 s\},
$$

for all $(x, r),(y, s) \in \mathbf{B} X$.

Now we are in position of establishing our main result.

Theorem 1. Let $(X, p)$ be partial metric space and let $P^{\prime}$ be the partial metric on $\mathbf{B} X$ defined above. Then

(a) The mapping $i: X \rightarrow \mathbf{B} X$ given by $i(x)=(x, 0)$, is an isometry

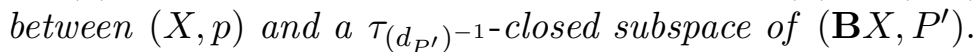

(b) $\leq_{P^{\prime}}=\sqsubseteq_{d_{p}}$, and $i\left(\operatorname{Max}\left(X, \leq_{p}\right)\right)=\operatorname{Max}(\mathbf{B} X)$.

(c) If $(X, p)$ is complete, then $\tau_{P^{\prime}}=\sigma(\mathbf{B} X)$.

(d) If $(X, p)$ is complete and $\operatorname{ker} p=\operatorname{Max}\left(X, \leq_{p}\right)$, then $\sigma(\mathbf{B} X)_{\mid \operatorname{Max}(\mathbf{B} X)}=$ $\lambda(\mathbf{B} X)_{\mid \operatorname{Max}(\mathbf{B} X)}$.

(e) $\left(\mathbf{B} X, P^{\prime}\right)$ is complete if and only if $(X, p)$ is complete.

Proof. (a) Let $x, y \in X$. Then

$$
P^{\prime}(i(x), i(y))=P^{\prime}((x, 0),(y, 0))=\max \{p(x, y), p(x, x), p(y, y)\}=p(x, y) .
$$

So $i$ is an isometry from $(X, p)$ into $\left(\mathbf{B} X, P^{\prime}\right)$.

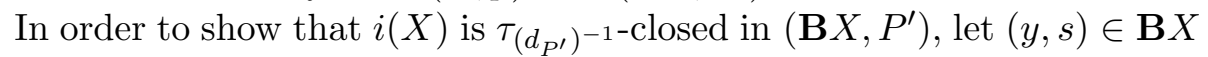
such that there is a sequence $\left(x_{n}\right)_{n \in \mathbb{N}}$ in $X$ with $d_{P^{\prime}}\left(\left(x_{n}, 0\right),(y, s)\right)<1 / n$ for all $n \in \mathbb{N}$. It follows that $p\left(x_{n}, y\right)+s-p\left(x_{n}, x_{n}\right)<1 / n$, so $s<p\left(x_{n}, x_{n}\right)-$ $p\left(x_{n}, y\right)+1 / n \leq 1 / n$ for all $n \in \mathbb{N}$, and hence $s=0$. We conclude that

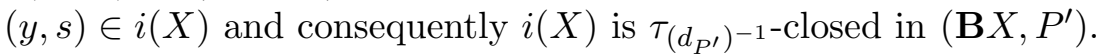

(b) For each $(x, r),(y, s) \in \mathbf{B} X$ we have

$$
\begin{aligned}
(x, r) & \leq P^{\prime}(y, s) \Leftrightarrow P^{\prime}((x, r),(y, s))=P^{\prime}((x, r),(x, r)) \\
& \Leftrightarrow p(x, y)+r+s \leq p(x, x)+2 r \\
& \Leftrightarrow p(x, y)-p(x, x) \leq r-s \Leftrightarrow(x, r) \sqsubseteq d_{p}(y, s) .
\end{aligned}
$$

So $\leq_{P^{\prime}}=\sqsubseteq d_{p}$.

On the other hand, it is clear (compare Theorem 5.1 of [16]) that $\operatorname{Max}(\mathbf{B} X)=$ $\left\{(x, 0): x \in \operatorname{Max}\left(X, \leq_{p}\right)\right\}$. Hence $i\left(\operatorname{Max}\left(X, \leq_{p}\right)\right)=\operatorname{Max}(\mathbf{B} X)$.

(c) If $(X, p)$ is complete, we have that $\left(\mathbf{B} X, \sqsubseteq d_{p}\right)$ is a domain by Theorem 3.1 of [16], and hence the sets $\Uparrow(x, r),(x, r) \in \mathbf{B} X$, form a base for $\sigma(\mathbf{B} X)$. Moroever, by Corollary 3.1 of [16], we have that $(x, r) \ll(y, s) \Leftrightarrow$ $d_{p}(x, y)<r-s$, for all $(x, r),(y, s) \in \mathbf{B} X$. 
It is easy to check that for each $(x, r) \in \mathbf{B} X$ and each $\varepsilon>0$, one obtains

$$
\Uparrow\left(x, r+\frac{\varepsilon}{2}\right) \subseteq B_{P^{\prime}}((x, r), \varepsilon) .
$$

Consequently $\tau_{P^{\prime}} \subseteq \sigma(\mathbf{B} X)$.

Now let $(z, t) \in \mathbf{B} X$ such that $(x, r) \in \Uparrow(z, t)$. Then, there is $\varepsilon>0$ such that $d_{p}(z, x)<t-r-\varepsilon$. Then, for $(y, s) \in B_{P^{\prime}}((x, r), \varepsilon)$, we have

$$
d_{p}(z, y) \leq d_{p}(z, x)+d_{p}(x, y)<(t-r-\varepsilon)+(r-s+\varepsilon)=t-s,
$$

and thus $(y, s) \in \Uparrow(z, t)$. Therefore $\sigma(\mathbf{B} X) \subseteq \tau_{P^{\prime}}$.

(d) It suffices to show that $\omega(\mathbf{B} X)_{\mid \operatorname{Max}(\mathbf{B} X)} \subseteq \sigma(\mathbf{B} X)_{\mid \operatorname{Max}(\mathbf{B} X)}$. Let $(x, r) \in$ $\operatorname{Max}(\mathbf{B} X)$ and $(z, t) \in \mathbf{B} X$ such that $(x, r) \in \mathbf{B} X \backslash \uparrow(z, t)$. By (b), $x \in \operatorname{Max}\left(X, \leq_{p}\right)$ and $r=0$. Then $p(x, x)=0$ by our hypothesis. Since $d_{p}(z, x)>t$ there exists $\varepsilon>0$ such that $d_{p}(z, x)>t+\varepsilon$, so, $B_{P^{\prime}}((x, 0), \varepsilon) \in$ $\sigma(\mathbf{B} X)$ by (c). Then, for each $(y, 0) \in B_{P^{\prime}}((x, 0), \varepsilon) \cap \operatorname{Max}(\mathbf{B} X)$, we have $p(y, y)=0$ and thus

$$
t+\varepsilon<d_{p}(z, x) \leq d_{p}(z, y)+d_{p}(y, x)=d_{p}(z, y)+d_{p}(x, y)<d_{p}(z, y)+\varepsilon .
$$

Hence $d_{p}(z, y)>t$, i.e., $(y, 0) \in \mathbf{B} X \backslash \uparrow(z, t)$. We conclude that $\omega(\mathbf{B} X)_{\mid \operatorname{Max}(\mathbf{B} X)} \subseteq$ $\sigma(\mathbf{B} X)_{\mid \operatorname{Max}(\mathbf{B} X)}$, so $\sigma(\mathbf{B} X)_{\mid \operatorname{Max}(\mathbf{B} X)}=\lambda(\mathbf{B} X)_{\mid \operatorname{Max}(\mathbf{B} X)}$.

(e) The proof is similar to the one given in Theorem 4.1 (e) of [16], so we omit some details.

Suppose that $\left(\mathbf{B} X, P^{\prime}\right)$ is complete and let $\left(x_{n}\right)_{n \in \mathbb{N}}$ be a Cauchy sequence in the metric space $\left(X,\left(d_{p}\right)^{s}\right)$. Then $\left(\left(x_{n}, 0\right)\right)_{n}$ is a Cauchy sequence in the complete metric space $\left(\mathbf{B} X,\left(d_{P^{\prime}}\right)^{s}\right)$, so there is $(z, t) \in \mathbf{B} X$ such that $\left(\left(x_{n}, 0\right)\right)_{n}$ converges to $(z, t)$ in $\left(\mathbf{B} X,\left(d_{P^{\prime}}\right)^{s}\right)$. So, in particular, we obtain that for each $\varepsilon>0, p(z, z)+2 r-p(z, z)<\varepsilon$, and thus $r=0$. Then, it immediately follows that $\left(x_{n}\right)_{n}$ converges to $z$ in $\left(X,\left(d_{p}\right)^{s}\right)$. We conclude that $p$ is a complete partial metric on $X$.

Conversely, suppose that $(X, p)$ is complete and let $\left(\left(x_{n}, r_{n}\right)\right)_{n \in \mathbb{N}}$ be a Cauchy sequence in $\left(\mathbf{B} X,\left(d_{P^{\prime}}\right)^{s}\right)$. Then, for each $\varepsilon>0$ there is $n_{\varepsilon} \in \mathbb{N}$ such that $p\left(x_{n}, x_{m}\right)-p\left(x_{n}, x_{n}\right)+r_{m}-r_{n}<\varepsilon$ and $p\left(x_{n}, x_{m}\right)-p\left(x_{m}, x_{m}\right)+r_{n}-r_{m}<$ $\varepsilon$ for all $n, m \geq n_{\varepsilon}$. Hence the sequence $\left(r_{n}\right)_{n \in \mathbb{N}}$ is bounded, so there exist a subsequence $\left(r_{n_{k}}\right)_{k \in \mathbb{N}}$ of $\left(r_{n}\right)_{n \in \mathbb{N}}$ and an $r \in \mathbb{R}^{+}$such that $\lim _{k} r_{n_{k}}=r$. It immediately follows that $\left(x_{n_{k}}\right)_{k \in \mathbb{N}}$ is a Cauchy sequence in $\left(X,\left(d_{p}\right)^{s}\right)$. Then, there is $x \in X$ such that $\left(x_{n_{k}}\right)_{k \in \mathbb{N}}$ converges to $x$ in $\left(X,\left(d_{p}\right)^{s}\right)$. Finally, it is easy to deduce that $\left(\left(x_{n}, r_{n}\right)\right)_{n \in \mathbb{N}}$ converges to $(x, r)$ in $\left(\mathbf{B} X,\left(d_{P^{\prime}}\right)^{s}\right)$. This concludes the proof. 
Remark 1. If $(X, p)$ is partial metric space such that $\tau_{p}$ is a $T_{1}$-topology, then $\operatorname{Max}\left(X, \leq_{p}\right)=X$, so, by Theorem $1(\mathrm{~b}), i(X)=\operatorname{Max}(\mathbf{B} X)$ in this case.

The following example shows that condition "ker $p=\operatorname{Max}\left(X, \leq_{p}\right)$ " can not be omitted in Theorem 1 (d).

Example 1. Let $X=\{0, \infty\} \cup \mathbb{N} \backslash\{1\}$, and let $p: X \times X \rightarrow \mathbb{R}^{+}$defined as $p(0,0)=0, p(0, \infty)=2, p(\infty, \infty)=0, p(n, n)=1 / n, p(0, n)=1+1 / n$, and $p(n, \infty)=1$ for all $n \in \mathbb{N} \backslash\{1\}$, and $p(n, m)=1+1 / n+1 / m$ for all $n, m \in \mathbb{N} \backslash\{1\}$ with $n \neq m$.

It is routine to check that $p$ is a partial metric on $X$, and it is complete because every Cauchy sequence in $\left(X,\left(d_{p}\right)^{s}\right)$ is eventually constant.

On the other hand, $\operatorname{Max}\left(X, \leq_{p}\right)=X$, so $i(X)=\operatorname{Max}(\mathbf{B} X)$ by Theorem 1 (b). However ker $p=\{\infty\}$.

We show that $\sigma(\mathbf{B} X)_{\mid \operatorname{Max}(\mathbf{B} X)} \neq \lambda(\mathbf{B} X)_{\mid \operatorname{Max}(\mathbf{B} X)}$. Indeed, we have $(0,0) \in$ $\mathbf{B} X \backslash \uparrow(\infty, 1)$ because $d_{p}(\infty, 0)=p(\infty, 0)-p(\infty, \infty)=2$. Nevertheless, for each $n \in \mathbb{N}$ we have

$$
(n+1,0) \in B_{P^{\prime}}((0,0), 1 / n) \cap \uparrow(\infty, 1),
$$

because $P^{\prime}((0,0),(n+1,0))=1+1 /(n+1)<1+1 / n=P^{\prime}((0,0),(0,0))+1 / n$, and $d_{p}(\infty, n+1)=1$ for all $n \in \mathbb{N}$.

Since by Theorem 1 (c), $\left\{B_{P^{\prime}}((0,0), 1 / n): n \in \mathbb{N}\right\}$ is a $\sigma(\mathbf{B} X)$-local base at $(0,0)$, we conclude that $\lambda(\mathbf{B} X)_{\mid \operatorname{Max}(\mathbf{B} X)}$ is strictly finer than $\sigma(\mathbf{B} X)_{\mid \operatorname{Max}(\mathbf{B} X)}$.

The computational model suggested by conditions (1)-(3) in p. 59 of [22] joint with Definition 5.1 of [16] and Theorem 1 above, motivate the following notions.

Definition 1. A partially metrizable computational model is a triple $(L, \sqsubseteq, P)$ such that $(L, \sqsubseteq)$ is a domain and $P$ is a complete partial metric on $L$ that induces the Scott topology.

Definition 2. A topological space $(X, \tau)$ has a partially metrizable computational model if there is a partially metrizable computational model $(L, \sqsubseteq, P)$ and an homeomorphism $\phi$ between $(X, \tau)$ and a $\tau_{\left(d_{P}\right)^{-1} \text {-closed sub- }}$ set of $(L, P)$ such that $\phi\left(\operatorname{Max}\left(X, \leq_{\tau}\right)\right)=\operatorname{Max}(L)$.

We conclude the paper with a characterization of those topological spaces that admit a compatible complete partial metric via the model proposed in 
Definition 1.

Theorem 2. A topological space has a partially metrizable computational model if and only if it admits a compatible complete partial metric.

Proof. Suppose that the topological space $(X, \tau)$ has a partially metrizable computational model $(L, \sqsubseteq, P)$. Let $\phi$ an homeomorphism between

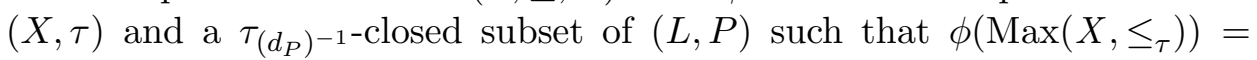
$\operatorname{Max}(L)$. For each $x, y \in X$ put $p(x, y)=P(\phi(x), \phi(y))$. Then $p$ is a partial metric on $X$ such that $\tau_{p}=\tau$. Moreover $p$ is complete: Indeed, let $\left(x_{n}\right)_{n \in \mathbb{N}}$ be a Cauchy sequence in the metric space $\left(X,\left(d_{p}\right)^{s}\right)$. Then $\left(\phi\left(x_{n}\right)\right)_{n}$ is a Cauchy sequence in the complete metric space $\left(L,\left(d_{P}\right)^{s}\right)$, so there is $z \in L$ such that $\left(\phi\left(x_{n}\right)\right)_{n}$ converges to $z$ in $\left(L,\left(d_{P}\right)^{s}\right)$, and hence in $\left(L,\left(d_{P}\right)^{-1}\right)$.

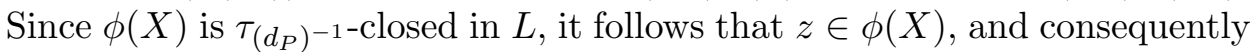
$\left(x_{n}\right)_{n}$ converges to $\phi^{-1}(z)$ in $\left(X,\left(d_{p}\right)^{s}\right)$. We conclude that $p$ is a complete partial metric on $X$.

Conversely, if $(X, \tau)$ admits a compatible complete partial metric $p$, then $\left(\mathbf{B} X, \sqsubseteq_{d_{p}}\right)$ is a domain. So, by Theorem $1,\left(\mathbf{B} X, \sqsubseteq_{d_{p}}, P^{\prime}\right)$ is a partially metrizable computational model for $(X, \tau)$.

\section{References}

[1] M. Ali-Akbari, B. Honari, M. Pourmahdian, M.M. Rezaii, The space of formal balls and models of quasi-metric spaces, Math. Struct. Comput. Sci. 19 (2009), 337-355.

[2] A. Edalat, R. Heckmann, A computational model for metric spaces, Theoret. Comput. Sci. 193 (1998), 53-73.

[3] A. Edalat, Ph. Sünderhauf, Computable Banach spaces via domain theory, Theoret. Comput. Sci. 219 (1999), 169-184.

[4] R. Engelking, General Topology, Monografie Mat. Tom 60, PWN-Polish Scientific Publishers, Warszawa, 1977.

[5] B. Flagg, R. Kopperman, Computational models for ultrametric spaces, Electronic Notes Theoret. Comput. Sci. 6 (1997), 151-159.

[6] G. Gierz, K.H. Hofmann, K. Keimel, J.D. Lawson, M. Mislove, D.S. Scott, Continuous Lattices and Domains, Encyclopedia of Mathematics and its Applications vol. 93. Cambridge Univ. Press, 2003. 
[7] R. Heckmann, Approximation of metric spaces by partial metric spaces, Appl.Cat. Struct. 7 (1999), 71-83.

[8] R. Kopperman, H.P. Künzi, P. Waszkiewicz, Bounded complete models of topological spaces, Topology Appl. 139 (2004), 285-297.

[9] M. Krötzsch, Generalized ultrametric spaces in quantitative domain theory, Theoret. Comput. Sci. 368 (2006), 30-49.

[10] H.P.A. Künzi, Nonsymmetric distances and their associated topologies: About the origins of basic ideas in the area of asymmetric topology, in: C.E. Aull, R. Lowen (Eds.), Handbook of the History of General Topology, vol. 3, Kluwer, Dordrecht, 2001, pp. 853-968.

[11] J. Lawson, Spaces of maximal points, Math. Struct. Comput. Sci. 7 (1997), 543-556.

[12] K. Martin, Domain theoretic models of topological spaces, Electronic Notes Theoret. Comput. Sci. 13 (1998), 173-181.

[13] S.G. Matthews, Partial metric topology, in: Procedings 8th Summer Conference on General Topology and Applications, Ann. New York Acad. Sci. 728 (1994), 183-197.

[14] J. Rodríguez-López, S. Romaguera, O. Valero, Denotational semantics for programming languages, balanced quasi-metrics and fixed points, Internat. J. Comput. Math. 85 (2008), 623-630.

[15] S. Romaguera, On Computational Models for the Hyperspace, Advances in Mathematics Research, volume 8, Nova Science Publishers, New York, 2009, pp. 277-294.

[16] S. Romaguera, O. Valero, A quantitative computational model for complete partial metric spaces via formal balls, Math. Struct. Comput. Sci. 19 (2009), 541-563.

[17] S. Romaguera, O. Valero, Domain theoretic characterisations of quasimetric completeness in terms of formal balls, Math. Struct. Comput. Sci. 20 (2010), 453-472.

[18] S. Romaguera, O. Valero, Internat. J. Comput. Math.

[19] J.J.M.M. Rutten, Weighted colimits and formal balls in generalized metric spaces, Topology Appl. 89 (1998), 179-202. 
[20] M. Schellekens, A characterization of partial metrizability. Domains are quantifiable, Theoret. Comput. Sci. 305 (2003), 409-432.

[21] M.B.Smyth, The constructive maximal point space and partial metrizability, Ann. Pure Appl. Logic 137 (2006), 360-379.

[22] P. Waszkiewicz, Quantitative continuous domains, Appl. Cat. Struct. 11 (2003), 41-67.

[23] P. Waszkiewicz, Partial metrisability of continuous posets, Math. Struct. Comput. Sci. 16 (2006), 359-372.

S. Romaguera and P. Tirado

Instituto Universitario de Matemática Pura y Aplicada

Universitat Politècnica de València

46022 Valencia, Spain

E-mail:sromague@mat.upv.es, pedtipe@mat.upv.es

O. Valero

Departamento de Ciencias Matemáticas e Informática

Universidad de las Islas Baleares

07111 Palma de Mallorca, Spain

E-mail: o.valero@uib.es 